\title{
Patterns in Listings of Failure-Rate \& MTTF Values and Listings of Other Data
}

\author{
Becker, Peter W.
}

Published in:

I E E E Transactions on Reliability

Link to article, DOI:

10.1109/TR.1982.5221273

Publication date:

1982

Document Version

Publisher's PDF, also known as Version of record

Link back to DTU Orbit

Citation (APA):

Becker, P. W. (1982). Patterns in Listings of Failure-Rate \& MTTF Values and Listings of Other Data. I E E E Transactions on Reliability, R-31(2), 132-134. https://doi.org/10.1109/TR.1982.5221273

\section{General rights}

Copyright and moral rights for the publications made accessible in the public portal are retained by the authors and/or other copyright owners and it is a condition of accessing publications that users recognise and abide by the legal requirements associated with these rights.

- Users may download and print one copy of any publication from the public portal for the purpose of private study or research.

- You may not further distribute the material or use it for any profit-making activity or commercial gain

- You may freely distribute the URL identifying the publication in the public portal

If you believe that this document breaches copyright please contact us providing details, and we will remove access to the work immediately and investigate your claim. 


\section{Patterns in Listings of Failure-Rate \& MTTF Values and Listings of Other Data}

\section{Peter W. Becker, Member IEEE \\ Technical University of Denmark, Lyngby}

\author{
Key Words-Failure rate values, MTTF values, Logarithmic distribu- \\ tion. \\ Reader Aids- \\ Purpose: Discuss an observation \\ Special math needed for explanations: Geometry and probability theory \\ Special math needed for results: Same \\ Results useful to: Analysts concerned with tables of MTTF and failure \\ rate values
}

\begin{abstract}
It has been observed that the decimal parts of failure-rate and MTTF values as listed in tables tend to have a logarithmic distribution. A possible explanation for this phenomenon is given. When such tables have been generated they should be examined to see if the anticipated distribution is present; should that not be the case, a systematic error might well be present. Such testing is one practical application of the observation.

The decimal-values from long lists of data quite often tend to have a logarithmic distribution as pointed out by Newcomb and Benford. The phenomenon may be explained in several different ways, depending upon the nature of the data. References to their papers and those by other authors are given. The reader may turn to tables in his own field of interest; such tables will in all likelihood also show the same regularity.
\end{abstract}

\section{INTRODUCTION}

While going over a listing of 79 failure rates [1] I noticed a surprising pattern; later going over a listing of 45 MTTF-values [2] I noticed the same pattern. Let us first discuss the failure rate values. If one takes a list of failure rate values for representative components and subsystems the numerical values will span several decades; in such a listing one will notice the following pattern: when the numerical value of a failure rate value is read from left to right the first non-zero digit (which is $1,2, \ldots, 8,9$ ) has a low value with much higher frequency than a high value! (All failure rate values are assumed given with at least 2-digit precision). This regularity does not seem to have been observed before by people working with reliability data. In [1] containing 79 values, " 1 or 2 " is the first nonzero digit in 40 cases, i.e. more than $50 \%$ of the 79 cases; " 7,8 , or 9" is the first non-zero digit in only 8 cases. In [2] containing 45 values, " 1 or 2 " is the first digit in 22 cases, " 7,8 , or 9" the first digit in 6 cases. The observations surprised me who naively had anticipated that, say, 2 and 8 would have been the first digit with about the same frequency because there are precisely as many rational numbers between 2 and 3 as between 8 and 9. (This phenomenon has nothing to do with "flinching" where the numbers people put down are influenced by their likes and dislikes for certain digits). As will be explained in the following, the above-mentioned observation plus some reflections have led me to conjecture that representative data from failure rate or MTTF tables tend to have logarithmically distributed decimal values-or what amounts to the same: the mantissae tend to have flat pdf's. Definitions of the terms are given below. In the following, a reason why these man-made devices show such a pattern is discussed. First, however, some possible explanations are presented as to why a similar pattern is encountered in tables of data of the most varied nature.

\section{Definitions}

To avoid confusion the following definitions will be used. Let the positive number $x$ have the representation: $x$ $\equiv d \cdot 10^{p}, 1 \leqslant d<10, p$ being any integer or zero, and $m \equiv$ $\log _{10} d$, then, in this paper $d, p$, and $m$ will be referred to as the decimal, the exponent, and the mantissa. The pdf is the probability density function.

\section{ARITHMETIC AND GEOMETRIC SERIES}

At this point it is appropriate to mention two different ways of illustrating numbers. First, as is commonly done, one can illustrate numbers as points on a line where larger numbers are illustrated by points further to the right on the line. The increments can be constant as on a ruler, illustrating numbers from an arithmetric series; or the increments can change as seen on semi-log paper where numbers from a geometric series are depicted as points with the same separation in the horizontal direction. Also one can use both a linear and a logarithmic scale on the same axis; if so, a point on the axis illustrates corresponding values of two variables, say, $m$ and $d$, if $m \equiv \log d$. Numbers from geometric series are frequently used in factual literature and in the ordinary affairs of life; e.g. standard values for resistors, wire, and drill gauges, the magnitude scale of the astronomers, Fechner's law.

A second way of illustrating the numbers would be to let the decimal value of the number be illustrated by a point on a circle's periphery (and store the exponent in some manner); this is for instance done on the circular slide rule. If we look at the values $x$ of a stochastic variable $X$, the pdf $f(x)$ can have the form illustrated in figure 1 . The decimal-values of $x$ then have the $\operatorname{pdf}_{d} f(d)$, illustrated in figure 2. If the circular slide rule representation is used, ${ }_{d} f(d)$ can be depicted as shown in figure 3. Had all $x$-values been, e.g. five times larger, the pdf in figure 3 should be moved $\log 5$ in the clockwise direction showing that by now $d$-values near unity have become less likely. If we simply measure the arc from the point indicated by $d=1$ 


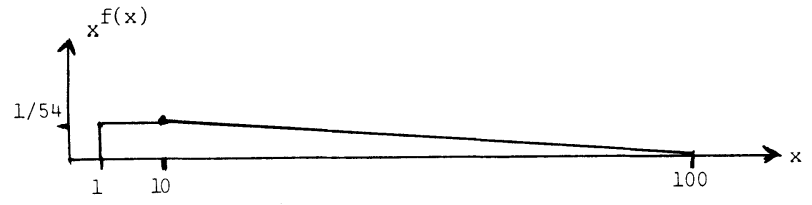

Fig. 1. Pdf for $x$, the values of the stochastic variable $X$. Linear scale is used on both axes.

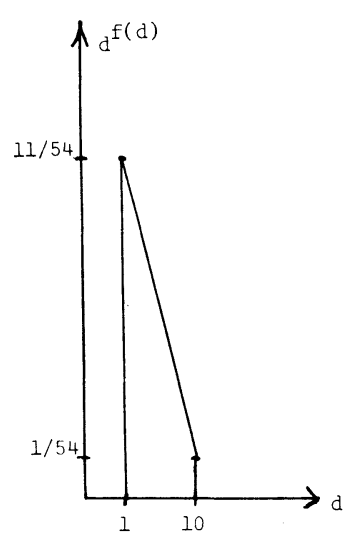

Fig. 2. Pdf for $d$, $d$ being the decimal part of the $x$-values. Linear scale is used on both axes.

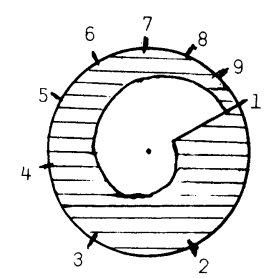

Fig. 3. Pdf of $d$ mapped on "circular slide rule". The length of the arc measured clockwise from 1 is $d$ using the non-linear scale indicated on the circle. If we instead measure the arc linearly using the periphery as our "unit of length" we obtain the value of $m=\log _{10} d$.

in the clockwise direction using the length of the circle's periphery as "unit of length", the length of the arc becomes $m=\log _{10} d$. The pdf (be it spiral-like or circular i.e. concentric with the circle) is valid for both $d$ and $m$ as long as $d$ - and $m$-values are measured using the proper scale on the periphery. The conjecture states that for representative failure rate values the pdf is circular; the same then automatically holds true for their reciprocals, the MTTF-values.

\section{OTHER TABLES SHOWING THE SAME PATTERN}

Having made the conjecture mentioned in the Introduction, I began to check the literature. Here it turned out that similar observations for tables with other kinds of data had been made in the past and explained in manners different from the one to be given for the failure rate values. Below in chronological order are listed some earlier explanations.

In 1881, Simon Newcomb [3] reported that the first pages of logarithmic tables wear out much faster than the last ones, whereas the pages of anti-logarithmic tables all seem to be used with the same intensity. About 50 years later Frank Benford [4] made the same observation. Newcomb next states that natural numbers basically can be considered as ratios of quantities. This point of view leads him to the conclusion that "the law of probability of the occurrence of numbers is such that all mantissae of their logarithms are equally probable'.

In 1938, Frank Benford published a study [4] showing that the above mentioned law holds for figures in tables of the most varied kinds, e.g. listings of areas of riversurfaces, street addresses for the first 342 people mentioned in the 1934 edition of American Men of Science. Benford referred to the observation as "the law of anomalous numbers". He explained the law as a natural phenomenon by claiming that Nature let phenomena fall into geometric series (as exemplified earlier), a view-if accepted-that explains the law.

In 1944, Goudsmit \& Furry came up with a different explanation [5]. They claim that the law "is merely the result of our way of writing numbers". The explanation is straightforward but hinges on how accurately a certain sum may be approximated by an integral; i.e. assuming that the approximation is a good one-and that must be checked in each case-the decimal-values $d$ are logarithmically distributed regardless of how $x$ is distributed as long as the $x$-values span many decades.

In 1970, R.W. Hamming examined the distribution of the mantissae of floating point numbers [6] and showed how the arithmetic operations of a computer transform various distributions towards the logarithmic distribution. An important property of the logarithmic distribution is its "persistence"; if $z$ equals $x / y$ or $x y$ where one of the decimals of $x$ or $y$ has the logarithmic distribution then $z$ will also get this distribution no matter how the other variable is distributed. The papers by Hafner \& Raimi [7, 8] are easy to read introductions to this little known corner of applied statistics.

\section{WHY THE DECIMALS OF FAILURE RATE VALUES HAVE A LOGARITHMIC DISTRIBUTION}

The following discussion is restricted to components for which the failure (hazard) rate, $\lambda$, is constant. As most electronic components are assumed to have a constant failure rate, the model is used extensively. For such components the failure rate and the Mean Time To Failure, MTTF, are reciprocal numbers. The results now to be discussed have to do with the numerical values of $\lambda$ 's and MTTFs for assorted components which represent the population of components used in the electrical and electronic industry. Each of the numerical values illustrates the compromise which (for that particular component) had to be made between cost, technology state-of-the-art, actual need for reliability, etc. The electrical and electronic components having the $79 \lambda$-values [1] were so chosen that they do represent components used in industry (the same holds true for the 45 MTTF-values [2]), i.e. the data illustrate a 
facet of the technology surrounding us just like Benford's listing of river-surface areas [4] illustrates a facet of the natural world. In all three cases we can scale the numerical values up or down at will by changing the unit, say, from hours to years, or from square miles to square kilometers. If the conversion factor has the value $h$, the new pdf for $d$ and $m$-as depicted in figure 3 -is obtained from the former one, by rotating it $\log _{10} h$ revolutions. Now, none of the units-chosen by man-differ in any essential respect from their scaled up or scaled down counterparts; this means that the pdf should keep its shape when rotated (if not there should be some deep meaning associated with certain ratios between proportional units-and that is obviously not the case). The only pdf which keeps its shape when rotated is the circular one; this implies that all $m$-values are equally probable and the $d$-values have the pdf ${ }_{d} f(d)=1 /\left(d \cdot \log _{e}(10)\right)$ and the distribution ${ }_{d} F(d)=$ $\log _{10} d$. We have thus demonstrated the conjecture stated in the Introduction. The invariance of the logarithmic distribution to change in scale has been mentioned in the literature by others.

Under the conjecture mentioned in the Introduction the decimal-value of a failure-rate figure will have 1 or 2 as the first digit with probability $\log 3-\log 1=0.477-0=$ $47.7 \%$ and 7,8 , or 9 as the first digit with probability $\log$ $10-\log 7=1-0.845=0.155=15.5 \%$; the two results $47.7 \%$ and $15.5 \%$ agree reasonably with the observed frequencies from [1]: 40/79 and 8/79. Also the MTTF frequencies from [2]: $22 / 45$ and $6 / 45$ are close to $47.7 \%$ and $15.5 \%$.

A practical application of the described pattern is that it might be used to quickly check lists of failure-rate or MTTF-values for systematic errors.

As an aside the reader can quickly verify for himself that even listings of street numbers from some column in a telephone directory-roughly speaking-have decimals with a logarithmic distribution.

\section{ACKNOWLEDGMENTS}

The papers listed below constitute fascinating reading, I am indebted to their authors. Also I would like to thank Dr. Marinus Kip for many helpful discussions.

\section{REFERENCES}

[1] P.W. Becker, F. Jensen, Design of Systems and Circuits for Maximum Reliability or Maximum Production Yield, McGraw-Hill, New York, 1977; Table A IV-1.

[2] S.R. Calabro, Reliability Principles and Practices, McGraw-Hill, New York, 1962; Table 7-2.

[3] S. Newcomb, "Note on the frequency of use of the different digits in natural numbers", Amer. J. Math., vol 4, 1881, pp 39-40.

[4] F. Benford, "The law of anomalous numbers", Proc. Amer. Phil. Soc., vol 78, pp 551-572.

[5] S.A. Goudsmit, W.F. Furry, "Significant figures of numbers in statistical tables", Nature, vol 154, 1944, pp 800-801.

[6] R.W. Hamming, "On the distribution of numbers", BSTJ, vol 49, 1970, pp 1609-1625.

[7] E. Hafner, "Circular slide roulette", IEEE Communications Magazine, vol 17, 1979 Mar, pp 29-31.

[8] R.A. Raimi, "The peculiar distribution of first digits", Scientific American, vol 221, 1969 Dec, pp 109-120.

\section{AUTHOR}

Peter Becker; Electronics Laboratory; Technical University of Denmark; DK-2800 Lyngby, DENMARK.

Dr. Becker (A'62, SM'78) received his MSc and dr.techn. degree in Electrical Engineering from the Technical University of Denmark, in 1950 and 1968 respectively. He has written several papers in the field of reliability. In 1977 he published the book "Design of Systems and Circuits for Maximum Reliability or Maximum Production Yield" (coauthored by Mr. Finn Jensen) with McGraw-Hill. In the same year Sovietskoy Radio published a Russian translation of the book; in 1978 it was republished by McGraw-Hill Kogakusha, Tokyo. He is also in terested in pattern recognition and has written a number of papers in that field. In 1968 he published the book "Recognition of Patterns Using the Frequencies of Occurrence of Binary Words"; a third, revised and enlarged edition appeared in 1978, published by Springer-Verlag. In 1972 he published the book "An Introduction to the Design of Pattern Recognition Devices" with Springer-Verlag. Dr. Becker is on the Editorial Board of the International Journal of General Systems. In connection with cultural agreements between Denmark and other nations he has given series of lectures in Cairo, Mexico City, and Peking.

Manuscript TR80-78 received 1980 June 30; revised 1981 November 2.

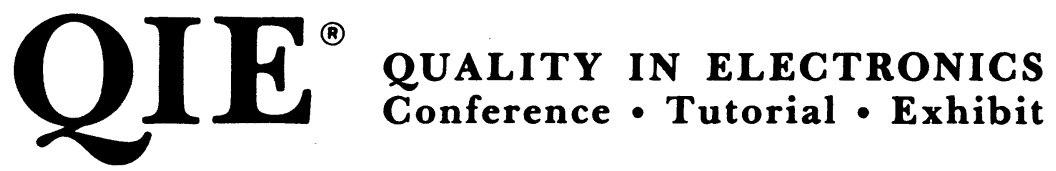

